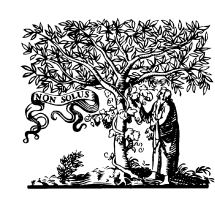

ELSEVIER

International Journal of Pressure Vessels and Piping 76 (1999) 49-53

\title{
Multiple crack problems for torsion thin-walled cylinder
}

\author{
Y.Z. Chen* \\ Laboratory for Nonlinear Mechanics of Continuous Media, Institute of Mechanics, Chinese Academy of Sciences, Beijing, 100080, People's Republic of China
}

Received 14 August 1998; accepted 24 August 1998

\begin{abstract}
Multiple crack problems for a torsion thin-walled cylinder are studied in this article. The boundary value problem is reduced to the solution of a Dirichlet problem of the Laplace equation. For the considered doubly connected region, a constant $k$ is involved in the boundary condition which is determined by the single-valuedness condition of the warping function. The finite difference method is suggested to solve the Dirichlet problem. The torsion rigidity coefficient can be evaluated numerically. The inverse of the torsion rigidity coefficient is known as the compliance coefficient. With the knowledge of the compliance coefficient, the stress intensity factor (SIF) can be evaluated. Numerical examples are given to demonstrate the use of the proposed method. Interaction effects between the cracks are also investigated. CC 1999 Elsevier Science Ltd. All rights reserved.
\end{abstract}

Keywords: Finite difference methods; Numerical solution procedures; Fracture mechanics; Stress intensity factor

\section{Introduction}

Crack problems for the torsion bar have been studied by several investigators [1-3]. The torsion crack problem for a circular section bar was investigated by using the complex variable function method [1]. By using the integral equation approach, the torsion crack problem of a rectangular section was solved [2]. The harmonic function continuation technique was suggested to solve the torsion crack problem of a rectangular section [3]. It is felt that the previously suggested technique cannot be directly used to solve the multiple crack problem for a torsion thin-walled cylinder.

In this article, multiple crack problems for torsion thinwalled cylinder are investigated. The relevant boundary value problem is reduced to solve the Dirichlet problem of the Laplace equation. For the considered doubly connected region, a constant $k$ is involved in the boundary condition which is determined by the single-valuedness condition of the warping function. The finite difference method is suggested to solve the Dirichlet problem, and the torsion rigidity coefficient $J$ can be easily evaluated by using the numerical integration. The inverse of the torsion rigidity coefficient is known as the compliance coefficient $C(=1 / J)$, which is the function of the crack length $a$. With the knowledge of the function $C(\mathrm{a})$, the stress intensity factor (SIF) can be finally

\footnotetext{
* Permanent address: Division of Engineering Mechanics, Jiangsu University of Science and Technology, Zhenjiang, Jiangsu 212013, People's Republic of China.
}

evaluated. In this article, the method is referred to as the computing compliance method. Finally, numerical examples are given to demonstrate the proposed method.

\section{Analysis}

It is well known that, for the torsion problem with a section as shown in Fig. 1(a), the governing equation in terms of the conjugate harmonic function $\psi(x, y)$ takes the form $[4$, p. 171]

$$
\begin{aligned}
& \nabla^{2} \psi(x, y)=0, \quad\left(\text { with } \nabla^{2}[]=\partial^{2}[] / \partial x^{2}+\partial^{2}[] / \partial y^{2}\right), \\
& \left.\psi\right|_{L_{1}}=\left.\left(\left(x^{2}+y^{2}\right) / 2\right)\right|_{L_{1}}+k \\
& \left.\psi\right|_{L_{2}}=\left(\left(x^{2}+y^{2}\right) /\left.2\right|_{L_{2}}\right.
\end{aligned}
$$

where $L_{1}\left(L_{2}\right)$ denotes the inner (outer) boundary of the section, and $k$ is a constant involved in the solution for multiple-connected region case (Fig. 1(a)). The constant $k$ will be determined by the single-value condition of the warping function, and takes the form $[4,5]$

$$
\oint_{L} \frac{\partial \psi}{\partial n} \mathrm{~d} s=0, \quad \text { or } \quad \oint_{L_{1}} \frac{\partial \psi}{\partial n} \mathrm{~d} s=0,
$$

where $L$ is any closed path between the paths $L_{1}$ and $L_{2}$, and the direction for $(n)$ and $(s)$ has been indicated in Fig. 1(a). 

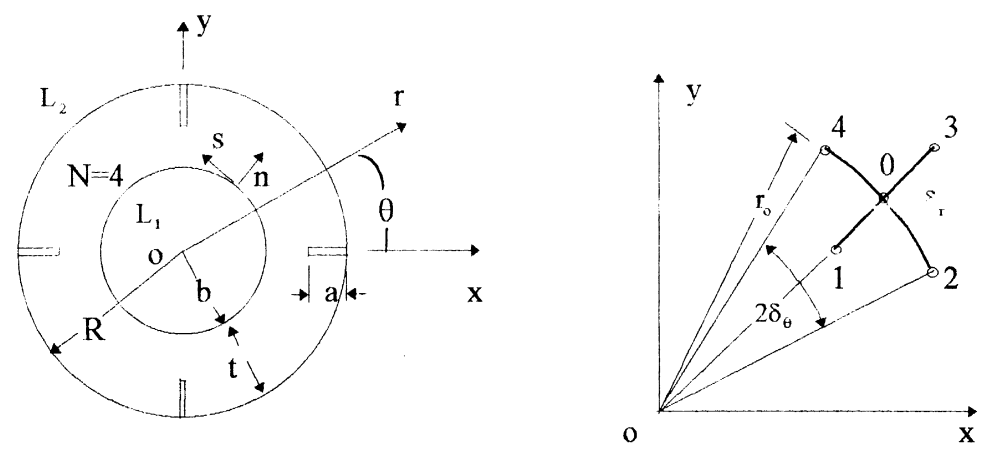

Fig. 1. (a) A thin-walled cylinder with multiple cracks at the outer boundary ( $N$ is the number of cracks). (b) The center node with adjacent nodes in the polar coordinate.

After the boundary value problem is solved, the torsion rigidity coefficient can be obtained as follows [4,5]

$J=\iint_{S}\left(2 \psi(x, y)-\left(x^{2}+y^{2}\right)\right) \mathrm{d} x \mathrm{~d} y+2 k \pi b^{2}$,

where $S$ denotes the region occupied by the cracked cylinder, and $b$ is the inner radius of the cylinder (Fig. 1(a)).

An appropriate solution technique was suggested previously [5] in which, we assumed

$\psi(x, y)=F(x, y)+K G(x, y)$.

In this case, the boundary value problem can be reduced to solve two individual problems for the functions $F(x, y)$ and $G(x, y)$

$\nabla^{2} F(x, y)=0$,

$\left.F\right|_{L_{1}}=\left(\left(x^{2}+y^{2}\right) / 2\right) \mid L_{1}$,

$\left.F\right|_{L_{2}}=\left.\left(\left(x^{2}+y^{2}\right) / 2\right)\right|_{L_{2}}$,

and

$\nabla^{2} G(x, y)=0$

$\left.G\right|_{L_{1}}=1$

$\left.G\right|_{L_{2}}=0$.

After the functions $F(x, y), G(x, y)$ are obtained from the numerical solution, substituting Eq. (5) into Eq. (3) yields $k=-c_{1} / c_{2}$,

where

$c_{1}=\oint_{L_{1}} \frac{\partial F}{\partial n} \mathrm{~d} s$

$c_{2}=\oint_{L_{1}} \frac{\partial G}{\partial n} \mathrm{~d} s$

Also, substituting Eq. (5) into Eq. (4)

$J=J_{1}+k J_{2}-\frac{\pi\left(R^{4}-b^{4}\right)}{2}+2 k \pi b^{2}$, where

$J_{1}=2 \iint_{S} F(x, y) \mathrm{d} y \mathrm{~d} y$,

$J_{2}=2 \iint_{S} G(x, y) \mathrm{d} y \mathrm{~d} y$.

To solve the boundary value problem shown by Eqs. (6), (7a) and (7b), the finite difference method is used. In fact, we solve the problem in the polar coordinate system $(r, \theta)$ (Fig. 1(b)). In this case, the Laplace equation (6) becomes

$\frac{\partial^{2} F}{\partial r^{2}}+\frac{1}{r} \frac{\partial F}{\partial r}+\frac{1}{r^{2}} \frac{\partial^{2} F}{\partial \theta^{2}}=0$.

From Eq. (14) we have the following approximation at the vicinity of the central node (Fig. 1(b))

$$
\begin{aligned}
& \frac{F_{1}-2 F_{0}+F_{3}}{\delta_{r}^{2}}+\frac{F_{3}-F_{1}}{2 r_{0} \delta_{t}}+\frac{F_{2}-2 F_{0}+F_{4}}{r_{o}^{2} \delta_{\theta}^{2}} \\
& \approx \frac{\partial^{2} F}{\partial r^{2}}+\frac{1}{r} \frac{\partial F}{\partial r}+\frac{1}{r^{2}} \frac{\partial^{2} F}{\partial \theta^{2}}=0
\end{aligned}
$$

(at the vicinity of central node) where $F_{0}, F_{1}, F_{2}, F_{3}, F_{4}$ denote the values of the function $F(x, y)$ at the relevant nodes (Fig. 1(b)). In addition, from Eq. (15) we have

$F_{0}=\left(\frac{F_{1}+F_{3}}{\delta_{r}^{2}}+\frac{F_{3}-F_{1}}{2 r_{0} \delta_{r}}+\frac{F_{2}+F_{4}}{r_{0}^{2} \delta_{\theta}^{2}}\right) /\left(\frac{2}{\delta_{r}^{2}}+\frac{2}{r_{0}^{2} \delta_{\theta}^{2}}\right)$

(at the vicinity of the central node). Therefore, an algebraic equation is obtainable, in which the node point values of the function $F(x, y)$ are the unknowns. The algebraic equation is solved by using the Siedel iteration procedure [6]. The boundary value problem shown by Eqs. (8), (9a) and(9b) can be solved in a similar manner. After the functions $F(x, y)$ and $G(x, y)$ are obtained from the numerical solution, the integrals (11a), (11b), (13a) and (13b) can also be evaluated numerically. Finally, the torsion rigidity coefficient $J$ is obtainable.

In this article, the inverse of the torsion rigidity coefficient is defined as the compliance coefficient $C=1 / J$. 


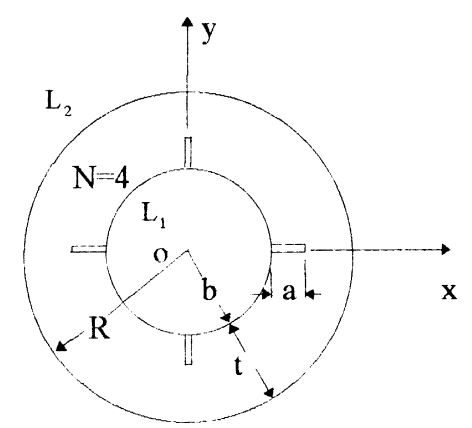

Fig. 2. A thin-walled cylinder with multiple cracks at the inner boundary.

In this case, $J$ and $C$ can be considered as functions of the crack length $a$. By using the computing compliance method, the SIFs at the crack tip can be expressed by [3]

$K_{3}=M \sqrt{\frac{1}{N} \frac{d}{d a} \frac{1}{J(a)}}=M \sqrt{\frac{1}{N} \frac{d C(a)}{d a}}$,

where $M$ is the torque applied at the end of cylinder, and $N$ the number of cracks.

For the cracked thin-walled cylinder with the inner cracks (Fig. 2), the relevant problem can be solved in a similar manner.

\section{Numerical examples}

Numerical examples are present in order to provide some new results, and to determine the interaction between cracks. In the case of cracks at the outer boundary, the boundary value problem is solved in the region $(0 \leq \theta \leq$ $\pi / N, b \leq r \leq R$ ) (Fig. 1(a)). The regions are divided into $40 \times 40$ meshes. The Seidel iteration method is used to solve the algebraic equation [6]. The computation is straightforward and will not be cited in detail. In the examples, the ratio $b / R$ is chosen as two cases 0.8 and 0.9 , the number of cracks is changed from $N=1,2,4$ to 10 , and the ratio alt is changed from $0.1,0.2$ to 0.9 .

The final calculated results for the torsional rigidity coefficient, the SIF at the crack tip are expressed, respectively, by:

$J=A(a / t, b / R, N) R^{4}$,

$K_{3}=B(a / t, b / R, N) M(R)^{-2.5}$,

where $M$ is the torque applied at the ends of the bar, and $N$ the number of cracks. The obtained numerical results for $A$

Table 1

Normalized torsion rigidity coefficient factor $A(a / t, b / R, N)$ for torsion a thin-walled cylinder with cracks at the outer boundary (see Fig. 1(a) and (19))

\begin{tabular}{|c|c|c|c|c|c|c|c|c|c|}
\hline$a / t$ & 0.1 & 0.2 & 0.3 & 0.4 & 0.5 & 0.6 & 0.7 & 0.8 & 0.9 \\
\hline \multicolumn{10}{|c|}{$b / R=0.8$} \\
\hline$N=1$ & 0.9254 & 0.9223 & 0.9181 & 0.9125 & 0.9053 & 0.8959 & 0.8834 & 0.8648 & 0.8267 \\
\hline$N=2$ & 0.9248 & 0.9199 & 0.9126 & 0.9026 & 0.8896 & 0.8728 & 0.8507 & 0.8200 & 0.7682 \\
\hline$N=4$ & 0.9236 & 0.9151 & 0.9018 & 0.8836 & 0.8600 & 0.8303 & 0.7925 & 0.7422 & 0.6662 \\
\hline$N=10$ & 0.9198 & 0.9004 & 0.8699 & 0.8292 & 0.7790 & 0.7200 & 0.6516 & 0.5714 & 0.4686 \\
\hline \multicolumn{10}{|c|}{$b / R=0.9$} \\
\hline$N=1$ & 0.5394 & 0.5383 & 0.5370 & 0.5352 & 0.5328 & 0.5297 & 0.5250 & 0.5170 & 0.4968 \\
\hline$N=2$ & 0.5392 & 0.5376 & 0.5354 & 0.5323 & 0.5284 & 0.5233 & 0.5163 & 0.5056 & 0.4828 \\
\hline$N=4$ & 0.5388 & 0.5364 & 0.5325 & 0.5272 & 0.5202 & 0.5110 & 0.4988 & 0.4815 & 0.4515 \\
\hline$N=10$ & 0.5379 & 0.5327 & 0.5243 & 0.5126 & 0.4974 & 0.4781 & 0.4535 & 0.4210 & 0.3725 \\
\hline
\end{tabular}

Table 2

Normalized SIFs $B(a / t, b / R, N)$ at the crack tip for torsion thin-walled cylinder with cracks at outer boundary (see Fig. 1(a) and (20))

\begin{tabular}{|c|c|c|c|c|c|c|c|c|c|}
\hline$a / t$ & 0.1 & 0.2 & 0.3 & 0.4 & 0.5 & 0.6 & 0.7 & 0.8 & 0.9 \\
\hline \multicolumn{10}{|c|}{$b / R=0.8$} \\
\hline$N=1$ & 0.3735 & 0.4628 & 0.5414 & 0.6213 & 0.7117 & 0.8265 & 1.0024 & 1.3934 & 1.8403 \\
\hline$N=2$ & 0.3254 & 0.4250 & 0.5102 & 0.5950 & 0.6881 & 0.8017 & 0.9607 & 1.2564 & 1.5911 \\
\hline$N=4$ & 0.2951 & 0.4042 & 0.4937 & 0.5803 & 0.6739 & 0.7871 & 0.9449 & 1.2225 & 1.5325 \\
\hline$N=10$ & 0.2803 & 0.3949 & 0.4882 & 0.5789 & 0.6763 & 0.7923 & 0.9502 & 1.2241 & 1.5300 \\
\hline \multicolumn{10}{|c|}{$b / R=0.9$} \\
\hline$N=1$ & 0.5400 & 0.6439 & 0.7437 & 0.8516 & 0.9852 & 1.1808 & 1.5193 & 2.3279 & 3.2186 \\
\hline$N=2$ & 0.4622 & 0.5743 & 0.6771 & 0.7829 & 0.9019 & 1.0556 & 1.2944 & 1.8327 & 2.4437 \\
\hline$N=4$ & 0.3912 & 0.5236 & 0.6352 & 0.7458 & 0.8674 & 1.0162 & 1.2249 & 1.6192 & 2.0653 \\
\hline$N=10$ & 0.3520 & 0.4923 & 0.6068 & 0.7183 & 0.8394 & 0.9859 & 1.1900 & 1.5485 & 1.9476 \\
\hline
\end{tabular}




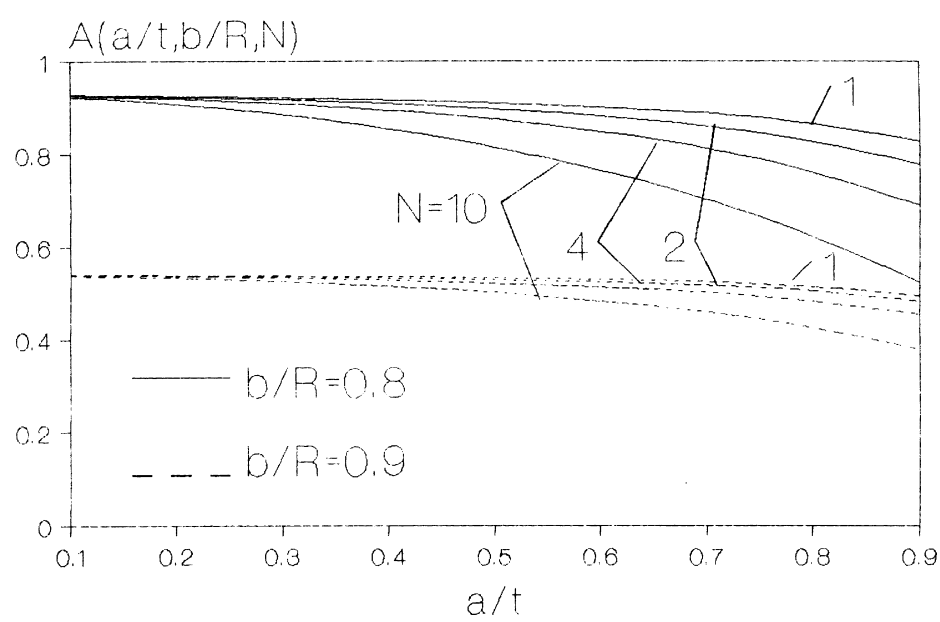

Fig. 3. Normalized torsion rigidity coefficients $A(a / t, b / R, N)$ (see Fig. 1 (a) and (19)).

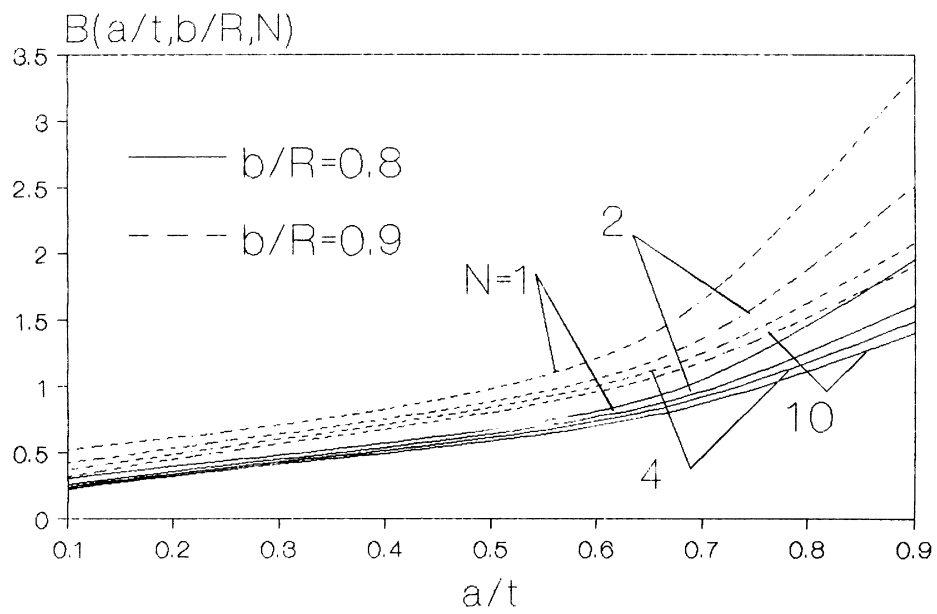

Fig. 4. Normalized SIFs $B(a / t, b / R, N)$ (see Figs. 1(a) and (20)).

$(a / t, b / R, N), B(a / t, b / R, N)$ are listed in Table 1 and Table 2 and in Fig. 3, and Fig. 4, respectively.

Interaction of cracks can be found from the present results. From Fig. 4 we see that, generally, in the same conditions of $a / t$ and $b / R$, if the number $(N)$ of cracks is larger, then the SIF at the crack tip becomes lower. This phenomenon was also observed in [7].

In the case of cracks at the inner boundary (Fig. 2), a

Table 3

Normalized torsion rigidity coefficient factor $D(a / t, b / R, N)$ for torsion thin-walled cylinder with cracks at inner boundary (see Fig. 2 and (21))

\begin{tabular}{|c|c|c|c|c|c|c|c|c|c|}
\hline$a / t$ & 0.1 & 0.2 & 0.3 & 0.4 . & 0.5 & 0.6 & 0.7 & 0.8 & 0.9 \\
\hline \multicolumn{10}{|c|}{$b / R=0.8$} \\
\hline$N=1$ & 0.9262 & 0.9240 & 0.9208 & 0.9163 & 0.9100 & 0.9015 & 0.8893 & 0.8700 & 0.8273 \\
\hline$N=2$ & 0.9258 & 0.9224 & 0.9170 & 0.9090 & 0.8980 & 0.8830 & 0.8622 & 0.8320 & 0.7780 \\
\hline$N=4$ & 0.9250 & 0.9191 & 0.9093 & 0.8951 & 0.8757 & 0.8499 & 0.8151 & 0.7667 & 0.6903 \\
\hline$N=10$ & 0.9224 & 0.9091 & 0.8871 & 0.8563 & 0.8164 & 0.7667 & 0.7057 & 0.6291 & 0.5239 \\
\hline \multicolumn{10}{|c|}{$b / R=0.9$} \\
\hline$N=1$ & 0.5396 & 0.5387 & 0.5375 & 0.5358 & 0.5336 & 0.5305 & 0.5258 & 0.5173 & 0.4954 \\
\hline$N=2$ & 0.5394 & 0.5380 & 0.5361 & 0.5334 & 0.5297 & 0.5248 & 0.5178 & 0.5069 & 0.4828 \\
\hline$N=4$ & 0.5392 & 0.5370 & 0.5337 & 0.5289 & 0.5224 & 0.5137 & 0.5018 & 0.4847 & 0.4540 \\
\hline$N=10$ & 0.5384 & 0.5339 & 0.5267 & 0.5163 & 0.5025 & 0.4845 & 0.4610 & 0.4291 & 0.3805 \\
\hline
\end{tabular}


Table 4

Normalized SIFs $E(t / a, b / R, N)$ at the crack tip for torsion thin-walled cylinder with cracks at inner boundary (see Fig. 2 and (22))

\begin{tabular}{|c|c|c|c|c|c|c|c|c|c|}
\hline$a / t$ & 0.1 & 0.2 & 0.3 & 0.4 & 0.5 & 0.6 & 0.7 & 0.8 & 0.9 \\
\hline \multicolumn{10}{|c|}{$b / R=0.8$} \\
\hline$N=1$ & 0.3085 & 0.3973 & 0.4791 & 0.5679 & 0.6698 & 0.8003 & 1.0016 & 1.4516 & 1.9559 \\
\hline$N=2$ & 0.2605 & 0.3609 & 0.4470 & 0.5363 & 0.6367 & 0.7598 & 0.9309 & 1.2529 & 1.6138 \\
\hline$N=4$ & 0.2402 & 0.3416 & 0.4267 & 0.5134 & 0.6100 & 0.7285 & 0.8933 & 1.1778 & 1.4909 \\
\hline$N=10$ & 0.2264 & 0.3285 & 0.4117 & 0.4939 & 0.5840 & 0.6933 & 0.8446 & 1.1088 & 1.4013 \\
\hline \multicolumn{10}{|c|}{$b / R=0.9$} \\
\hline$N=1$ & 0.5212 & 0.6089 & 0.7026 & 0.8195 & 0.9687 & 1.1824 & 1.5485 & 2.4129 & 3.3566 \\
\hline$N=2$ & 0.4224 & 0.5356 & 0.6384 & 0.7490 & 0.8765 & 1.0405 & 1.2957 & 1.8714 & 2.5187 \\
\hline$N=4$ & 0.3664 & 0.4889 & 0.5967 & 0.7096 & 0.8362 & 0.9912 & 1.2077 & 1.6194 & 2.0829 \\
\hline$N=10$ & 0.3204 & 0.4546 & 0.5653 & 0.6759 & 0.7978 & 0.9466 & 1.1540 & 1.5151 & 1.9142 \\
\hline
\end{tabular}

numerical solution can be obtained in a similar manner. As before, the final calculated results for the torsion rigidity the SIF are expressed by:

$J=D(a / t, b / R, N) R^{4}$,

$K_{3}=E(a / t, b / R, N) M(R)^{-2.5}$,

where $M$ is the torque applied at the ends of bar, and $N$ the number of cracks. The obtained numerical results for $D(a / t$, $b / R, N), E(a / t, b / R, N)$ are listed in Table 3 and Table 4 respectively. From Table 2 and Table 4 we see that, for the same conditions of $a / t, b / R$ and $N$, when alt $\leq 0.5$ the SIFs are slightly higher for the outer crack case.

\section{Remarks}

It is proved that the finite difference method for solving the boundary value problem and the computing compliance technique for evaluating the SIF provide an effective way to solve the torsion problem of a thin-walled cylinder with many cracks. The iteration for obtaining the solution of the algebraic equation is easy in computation. Also, as the computing compliance method was used to evaluate the
SIF, the demand for the function $F(x, y)$ and $G(x, y)$ at the vicinity of the crack tip is not serious.

\section{Acknowledgements}

The research project is supported by the National Natural Science Fund of China.

\section{References}

[1] Sih GC. Strength of stress singularities at crack tips for flexural and torsional problem. Journal of Applied Mechanics 1963;30:419-425.

[2] Westermann RA, Yang WH. Stress analysis of cracked rectangular beams. Journal of Applied Mechanics 1967;34:693-701.

[3] Chen YZ. Solutions of the torsion crack problems of a rectangular bar by using harmonic function continuation technique. Engineering Fracture Mechanics 1980;13:193-212.

[4] Sokolnikoff IS. Mathematical theory of elasticity, New York: McGraw-Hill, 1956.

[5] Chen YZ. Solutions of the torsion problem for some kinds of bars with multiply connected section. International Journal of Engineering Science 1983;21:813-823.

[6] Hildebland FB. Introduction to numerical analysis, New York: McGraw-Hill, 1974.

[7] Chen YZ, Hasebe N. New integration scheme for the branch crack problem. Engineering Fracture Mechanics 1995;52:791-801. 